\title{
CONVERSION FROM NONSTANDARD TO STANDARD MEASURE SPACES AND APPLICATIONS IN PROBABILITY THEORY
}

\author{
BY
}

\author{
PETER A. LOEB(1)
}

\begin{abstract}
Let $(X, a, \nu)$ be an internal measure space in a denumerably comprehensive enlargement. The set $X$ is a standard measure space when equipped with the smallest standard oalgebra $\pi$ containing the algebra $Q$, where the extended real-valued measure $\mu$ on $\pi$ is generated by the standard part of $\nu$. If $f$ is $Q$-measurable, then its standard part $0_{f}$ is $M$-measurable on $X$. If $f$ and $\mu$ are finite, then the $\nu$-integral of $f$ is infinitely close to the $\mu$-integral of ${ }^{0} f$. Applications include coin tossing and Poisson processes. In particular, there is an elementary proof of the strong Markov property for the stopping time of the $j$ th event and a construction of standard sample functions for Poisson processes.
\end{abstract}

1. Introduction. Let $(X, Q, \nu)$ be an internal measure space in a denumerably comprehensive enlargement (e.g., a suitable ultrapower) of a struc-

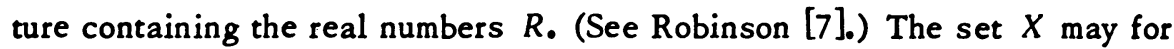
example be a *finite set, i.e., a set $\left\{x_{i}: 1 \leq i \leq \omega\right\}$ indexed by an initial segment of the nonstandard natural numbers ${ }^{*} N$, and $\nu$ may be given by a similarly indexed set of nonnegative numbers $\left\{a_{i}: 1 \leq i \leq \omega\right\}$ in the extension ${ }^{*} R$ of $R$ with $\nu(A)=\Sigma_{x_{i} \in A} a_{i}$ for each internal set $A \subset X$. In [4] we showed that a standard probability space can be transformed into such a *finite set and family of weights. We now show that if $(X, Q, \nu)$ is an arbitrary nonstandard measure space (and even if $\mathcal{G}$ is only an internal algebra and $\nu$ a ${ }^{*}$ finitely additive measure on $\mathfrak{Q}$ ), the set $X$ itself, considered now as a standard set, is a standard measure space when equipped with the smallest standard $\sigma$-algebra $\pi$ generated by $\mathcal{G}$ in $X$ with the measure $\mu$ on $\pi$ generated by the standard part ${ }^{0} \nu$ of the measure $\nu$ on $\mathcal{G}$. If $f: X \rightarrow{ }^{*} R$ is an internal

Presented to the Society, January 26, 1973 under the title An introduction to nonstandard analysis with applications to the generation of new "standard" sample spaces for probability theory; received by the editors May 12, 1974.

AMS (MOS) subject classifications (1970). Primary 02H25, 28A10, 60A05, 60J99.

Key words and phrases. Measure space, probability space, standard integral, coin tossing, Poisson processes, sample function, strong Markov property.

(1) This work was supported by NSF Grant GP 14785, and was presented at the 1974 Oberwolfach meeting on nonstandard analysis dedicated to the memory of Abraham Robinson. The author is indebted to Professors J. L. Doob and D. J. Brown for several helpful conversations. 
Q-measurable function, then its standard part $0 f$ is a standard M-measurable, extended real-valued function on $(X, \mathbb{M})$. If ${ }^{0} f$ and $\mu$ take only finite values, then for any $A \in \mathbb{Q}, \int_{A}{ }^{0} f d \mu$ and $\int_{A} f d \nu$ differ by only an infinitesimal. Applications of this theory include coin tossing and Poisson processes. In particular we give an elementary proof of the strong Markov property for the stopping time of the $j$ th event and a construction of standard sample functions for Poisson processes.

Recall that $a \simeq b$ in ${ }^{*} R$ means that $a-b$ is in the monad of 0 . Here, as in general, we use Robinson's notation [7] with the following exceptions: the symbol $\mu$ is used to denote a measure and $m(0)$ to denote the monad of zero; if $a \epsilon^{*} R$, then ${ }^{0} a$ denotes the unique real number $r$ with $a \simeq r$ if $a$ is finite (i.e., $|a| \leq n$ for some $n \in N$ ), while ${ }^{0} a=+\infty$ if $a \geq n$ for all $n \in N$ and ${ }^{0} a=-\infty$ if $a \leq-n$ for all $n \in N$. Here $N$ and ${ }^{*} N$ are the standard and nonstandard natural numbers; we assume that $0 \in N$ and set $1 / 0=+\infty$.

2. Construction of measure spaces. We begin with an internal set $X$ in a denumerably comprehensive enlargement. This means that if $S$ is a standard set and $A_{n}$ is internal with $A_{n} \epsilon^{*} S$ for each $n \in N$, then the external sequence $\left\{A_{n}: n \in N\right\}$ is the restriction to $N$ of an internal function from ${ }^{*} N$ into ${ }^{*} S$. Enlargements which are ultrapowers or $K_{\cdot 1}$ saturated models have this property. (See [6, pp. 27-35].) Of course by the set $X$ we mean the collection of internal objects $x \in{ }^{*} S$, for some standard set $S$, which satisfy the relation $x \in X$. Thus an external sequence $\left\{x_{n}: n \in N\right\}$ in $X$ is the restriction to $N$ of an internal "sequence" $\left\{x_{n}: n \in{ }^{*} N\right\}$ in ${ }^{*} S$.

Let $\mathcal{G}$ be an intemal collection of internal subsets of $X$. We need only assume that $\mathcal{G}$ is an algebra; that is, if $A$ and $B$ are in $\mathcal{G}$, then $A \cup B \in \mathbb{Q}$ and $X-A \in \mathbb{Q}$. Since the union of any two sets in $\mathcal{Q}$ is in $\mathcal{Q}$, it follows by internal induction over ${ }^{*} N$ that ${ }^{*}$ finite unions of sets in $\mathbb{Q}$ are again in $\mathcal{G}$. We now show, however, that countable infinite unions of disjoint nonempty sets in $\mathbb{Q}$ are not in $\mathfrak{Q}$.

Proposition 1. Given $A_{n} \in \mathbb{Q}$ for each $n \in N$, if $A_{0} \subset \bigcup_{n=1 ; n \in N}^{\infty} A_{n^{\prime}}$ then there is an $m \in N$ with $A_{0} \subset \bigcup_{n=1}^{m} A_{n}$.

Proof. Applying the preceding remarks to $\mathbb{Q}$ instead of $X$, we let $\left\{A_{n}\right.$ : $\left.n \in{ }^{*} N\right\}$ be an internal sequence extending the given sequence $\left\{A_{n}: n \in N\right\}$. Then the set $\left\{m \in \epsilon^{*} N: A_{0} \subset \bigcup_{n=1}^{m} A_{n}\right\}$ is internal, nonempty, and has a first element which must be finite.

Let $\nu$ be an internal mapping of $\mathcal{Q}$ into the nonnegative elements of ${ }^{*} R$, and assume that $\nu$ is finitely additive. That is, $\nu(\varnothing)=0$ and if $A$ and $B$ are 
sets in $\mathbb{Q}$ with $A \cap B=\varnothing$, then $\nu(A \cup B)=\nu(A)+\nu(B)$. For each $A \in \mathbb{Q}$, set $\mu(A)={ }^{0}(\nu(A)) ; \mu$ also is finitely additive and its range is in $R \cup\{+\infty\}$. Let $M$ denote the smallest collection of subsets of $X$, both internal and external, such that $\pi$ is a $\sigma$-algebra in the standard sense and $M \supset \mathcal{G}$. We apply Carathéodory's Extension Theorem [8, pp. 253-260] to show that $(X, M, \mu)$ is a standard measure space.

Theorem 1. The extended real-valued function $\mu$ has a standard, o-additive extension to the smallest (external) $\sigma$-algebra $M$ in $X$ containing $G$. For each $B \in M$, the value of this extension is given by $\mu(B)=\inf _{A \in Q, B \subset A} \mu(A)$. The extension is unique if $\mu(X)<+\infty$, in which case for each $B \in M, \mu(B)=$ $\sup _{A \in Q, B \supset A} \mu(A)$ and there is an $A \in \mathbb{Q}$ with $\mu((B-A) \cup(A-B))=0$. (2) $^{2}$

Proof. By Proposition 1, a countable, infinite union of disjoint nonempty sets in $\mathcal{G}$ cannot be an element of $\mathcal{G}$. We may, therefore, form an outer measure from $\mu$ and thus extend $\mu$ to $\mathbb{M}$. Given $B \in M$ with $\mu(B)<+\infty$ and given $\epsilon>0$ in $R$, there is a sequence $\left\{A_{n} \in \mathbb{G}: n \in N\right\}$ with $A_{n} \subset A_{n+1}$ for each $n \in N$ such that $B \subset C=\bigcup_{n=0 ; n \in N}^{\infty} A_{n}$ and $\mu(C)<\mu(B)+\epsilon_{0}$. Extend the given sequence to an internal sequence $\left\{A_{n}: n \epsilon^{*} N\right\}$, and choose $\omega \epsilon^{*} N-N$ so that if $1 \leq n \leq \omega$, then $A_{n-1} \subset A_{n}$ and $A_{n} \in \mathbb{G}$. We may also assume that $\nu\left(A_{\omega}\right)<\mu(B)+\epsilon_{0}$ Since $B \subset C \subset A_{\omega}$, it follows that $\mu(B)=\inf _{A \in \mathbb{Q}, B \subset A} \mu(A)$.

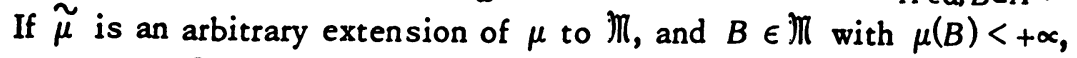
then given $A \in \mathbb{Q}$ and $\epsilon>0$ in $R$ with $B \subset A$ and $\mu(A)<\mu(B)+\epsilon$, we see

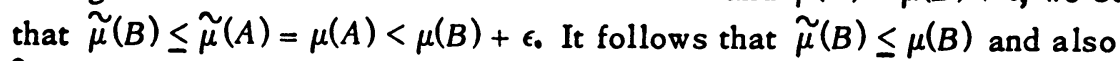
$\tilde{\mu}(A-B) \leq \mu(A-B)$, whence $\mu(A)=\tilde{\mu}(A)=\tilde{\mu}(A-B)+\tilde{\mu}(B) \leq \mu(A-B)+$ $\mu(B)=\mu(A)$. Thus we have the well-known fact that $\tilde{\mu}(B)=\mu(B)$ for each $B$ $\epsilon \mathbb{M}$ with $\mu(B)<+\infty$. The rest of the proof is clear.

Examples of spaces $(X, Q, \nu)$ can be obtained by taking the nonstandard extension in a denumerably comprehensive enlargement of a standard set $S$ equipped with an algebra $\mathcal{L}$ of subsets and a finitely additive measure $\lambda$ on $\mathfrak{L}$.(3) In the following examples, $\mathcal{L}$ is the collection of Lebesgue measurable

(2) (Added April 1975.) Ward Henson has proved the uniqueness of the extension when $\mu(X)=+\infty$. The proof uses the fact that each $B \in \Pi$ is in the $\sigma$-algebra generated by some countable algebra $Q^{\prime} \subset Q$ to show that either there is an $A \in Q$ with $A \subset B$ and $\mu(A)=+\infty$ or there is a sequence $\left\{A_{n} \in \mathbb{Q}: n \in N\right\}$ with $\mu\left(A_{n}\right)<+\infty$ for each $n$ and $B \subset \cup_{n \in N} A_{n}$.

(3) (Added August 1974.) In an unpublished manuscript written in 1966, J. J. Uhl, Jr. noted that $\lambda$ has a $\sigma$-additive extension to the external $\sigma$-algebra in ${ }^{*} S$ generated by the externalalgebra consisting of extensions of standard sets in $\mathcal{L}$. Though stated for a countable ultraproduct of $S$, the result is valid for any enlargement. For comparison with the Stone space of $L^{\infty}(\lambda)$, see $[4$, p. 77$]$. 
sets in $R$ and $\lambda$ is Lebesgue measure on $R$.

Example 1. Let $X={ }^{*} R, \mathscr{Q}={ }^{*} \mathscr{Q}$, and $\nu={ }^{*} \lambda$. Then $C=\bigcup_{n=1 ; n \in N}^{\infty}{ }^{*}[-n, n]$ is in $\mathbb{M}$ and $\mu(C)=+\infty$. Note that

$$
\bigcup_{n=1 ; n \in N}^{\infty} *[-n, n] \neq *\left(\bigcup_{n=1 ; n \in N}^{\infty}[-n, n]\right)=* R .
$$

The monad of zero, $m(0)$, is the $\prod_{\text {-measurable set }} \bigcap_{n=1 ; n \in N}^{\infty}{ }^{*}[-1 / n, 1 / n]$ and $\mu(m(0))=0$. Since $R$ is contained in a ${ }^{*}$ finite set in ${ }^{*} R, R$ has zero outer measure.

Example 2. Given $\omega \epsilon^{*} N-N$, let $X=[0, \omega] \subset{ }^{*} R, \mathbb{Q}=\left\{A \in \epsilon^{*} \mathcal{Q}: A \subset\right.$ $[0, \omega]\}$, and $\nu={ }^{*} \lambda / \omega$. Then $\mu(X)=1$ and $\mu\left(\bigcup_{n=1 ; n \in N}^{\infty}{ }^{*}[0, n]\right)=0$.

Example 3. Given $\omega \epsilon^{*} N-N$, let $X=\left\{n \epsilon^{*} N: 0 \leq n<\omega\right\}$ with addition $\oplus$ defined by setting $n \oplus m=n+m$ when $n+m<\omega$ and $n \oplus m=n+m-\omega$ when $n+m \geq \omega$. Let $\mathbb{Q}$ be the class of all intemal subsets of $X$. For each $A \in \mathbb{Q}$, set $\nu(A)=|A| / \omega$, where $|A|$ denoces the internal cardinality of $A$. Call elements $n$ and $m$ of $X$ equivalent if there is a standard $k \in N$ with either $n \oplus k=m$ or $m \oplus k=n$. A set $B$ which contains exactly one element from each equivalence class is not measurable since $X$ equals the disjoint union $\bigcup_{n=0 ; n \in N}^{\infty}((B \oplus n) \cup(B \oplus(\omega-n)))$.

We next show that $\mathcal{Q}$-measurable functions become $\prod_{\text {-measurable when we }}$ take their standard part on $X$. The standard part ${ }^{0} f$ of a function $f: X \rightarrow{ }^{*} R$ takes the value ${ }^{0}(f(x))$ at each $x \in X$. We say that $f$ is $Q_{\text {-measurable if } f \text { is }}$ internal and for each $a \in^{*} R,\{x \in X: f(x)<a\} \in \mathbb{Q}$ and $\{x \in X: f(x) \leq a\} \in \mathbb{G}$. Of course, we only need one of these conditions if $\mathcal{G}$ is an internal $\sigma$-algebra in the nonstandard sense, i.e., " $\sigma$ " refers to ${ }^{*} N$.

Theorem 2. If $f: X \rightarrow{ }^{*} R$ is $\mathbb{G}$-measurable, then $0 f: X \rightarrow R \cup\{+\infty,-\infty\}$ is M-measurable.

Proof. Given any standard $a \in R$,

$$
\left\{x \in X:{ }^{0} f(x)<a\right\}=\bigcup_{n=1 ; n \in N}^{\infty}\{x \in X: f(x)<a-1 / n\} \in \mathbb{M} .
$$

One can easily extend Theorem 2 to the case of an internal mapping $F$ from $X$ into the extension ${ }^{*} Z$ of a compact metric space $Z$ with metric $d$. Here we assume that for each point $z_{i}$ in some countable dense subset of $Z$ and for each standard rational number $q$, we have $\left\{x \in X:{ }^{*} d\left(z_{i}, F(x)\right)<q\right\} \in \mathbb{Q}$.

In converting nonstandard integrals into standard integrals, we need to consider the fact that the product of any infinite positive number $a$ with $1 / \alpha$ 
is 1 while ${ }^{0} \alpha \cdot{ }^{0}(1 / \alpha)=+\infty \cdot 0$. For this reason we assume that $\mu(X)<+\infty$ and we only consider $\mathbb{G}$-measurable functions $f$ taking values in the finite nonstandard real numbers. Recall, however, that for such an $f$, the set in $\epsilon$ $\left.{ }^{*} N: \forall x \in X,|f(x)| \leq n\right\}$ has a first element which must be finite. (See for example 3.3 .3 of [7].) In what follows, we use notation such as $f^{-1}[a, b)$ and ${ }^{0} f^{-1}[a, b)$ for the sets $\{x \in X: a \leq f(x)<b\}$ and $\left\{x \in X: a \leq{ }^{0} f(x)<b\right\}$.

Theorem 3. Assume that $\mu(X)<+\infty$, and let $f: X \rightarrow^{*}[-n, n], n \in N$, be G.measurable. Then, for each $A \in \mathbb{Q}, \int_{A} f d \nu \simeq \int_{A}^{0} f d \mu$.

Proof. We assume that $A=X$; the general case follows from the fact that ${ }^{0}\left(f \cdot \chi_{A}\right)={ }^{0} f \cdot \chi_{A}$ where $\chi_{A}(x)=1$ if $x \in A$ and $\chi_{A}(x)=0$ if $x \notin A$. We may also assume that for some $\delta>0$ in $R, f(x) \geq \delta$ for all $x \in X$; this follows from the fact that if, for $k=2 \delta+\sup _{x \in X}\left|{ }^{0} f(x)\right|$, we have $\int(f+k) d \nu$ $\simeq \int\left({ }^{0} f+k\right) d \mu$, then since $\int k d \nu \simeq \int k d \mu$, we have $\int f d \nu \simeq \int{ }^{0} f d \mu$. Let $D=$ $\left\{r \in R: \mu\left({ }^{0} f^{-1}[r]\right)>0\right\} ; D$ is finite or countably infinite in $R$. Let $M=\mu(X)+$ 1 and fix $\epsilon>0$ in $R$. There is an increasing finite sequence $0=y_{0}<y_{1}<$ $y_{2}<\cdots<y_{m}$ in $R$, with $y_{m}>\sup _{x \in X}{ }^{0} f(x)$, such that $y_{i} \notin D$ and $y_{i}-$ $y_{i-1}<\epsilon / 3 M$ for $1 \leq i \leq m$. Let

$$
\begin{array}{ll}
\underline{S}_{\nu}=\sum_{i=1}^{m} y_{i-1} \nu\left(f^{-1}\left[y_{i-1}, y_{i}\right)\right), & \bar{S}_{\nu}=\sum_{i=1}^{m} y_{i} \nu\left(f^{-1}\left[y_{i-1}, y_{i}\right)\right), \\
\underline{S}_{\mu}=\sum_{i=1}^{m} y_{i-1} \mu\left({ }^{0} f^{-1}\left[y_{i-1}, y_{i}\right)\right), & \bar{S}_{\mu}=\sum_{i=1}^{m} y_{i} \mu\left({ }^{0} f^{-1}\left[y_{i-1}, y_{i}\right)\right)
\end{array}
$$

Then

$$
\begin{gathered}
\underline{S}_{\nu} \leq \int_{X} f d \nu \leq \bar{S}_{\nu}, \quad \underline{S}_{\mu} \leq \int_{X} 0 f d \mu \leq \bar{S}_{\mu}, \\
\bar{S}_{\nu}-\underline{S}_{\nu} \leq \frac{\epsilon}{3 M} \sum_{i=1}^{m} \nu\left(f^{-1}\left[y_{i-1}, y_{i}\right)\right)<\frac{\epsilon}{3},
\end{gathered}
$$

and similarly, $\bar{S}_{\mu}-\underline{S}_{\mu}<\epsilon / 3$. For any $i, 1 \leq i \leq m,{ }^{0} f^{-1}\left(y_{i-1}, y_{i}\right) \subseteq$ $f^{-1}\left(y_{i-1}, y_{i}\right) \subseteq f^{-1}\left[y_{i-1}, y_{i}\right] \subseteq f^{-1}\left[y_{i-1}, y_{i}\right]$. Therefore,

$$
\begin{aligned}
\mu\left({ }^{0} f^{-1}\left[y_{i-1}, y_{i}\right]\right) & =\mu\left({ }^{0} f^{-1}\left[y_{i-1}, y\right)\right)=\mu\left({ }^{0} f^{-1}\left(y_{i-1}, y_{i}\right)\right) \\
& \leq \mu\left(f^{-1}\left(y_{i-1}, y_{i}\right)\right) \simeq \nu\left(f^{-1}\left(y_{i-1}, y_{i}\right)\right) \leq \nu\left(f^{-1}\left[y_{i-1}, y_{i}\right)\right) \\
& \leq \nu\left(f^{-1}\left[y_{i-1}, y_{i}\right]\right) \simeq \mu\left(f^{-1}\left[y_{i-1}, y_{i}\right]\right) \leq \mu\left({ }^{0} f^{-1}\left[y_{i-1}, y_{i}\right]\right) .
\end{aligned}
$$


It follows that $\underline{S}_{\nu} \simeq \underline{S}_{\mu}$, and thus $\left|\int_{X} f d \nu-\int_{X}{ }^{0} f d \mu\right|<\epsilon$. Since $\epsilon>0$ is arbitrary, we are done.

Corollary 1. Let $Y=\left\{y_{i}: 1 \leq i \leq \omega\right\}$ and $\left\{\beta_{i} \epsilon^{*} R: 1 \leq i \leq \omega\right\}$ be ${ }^{*}$ finite sets of the same internal cardinality in a denumerably comprehensive enlargement with $\beta_{i} \geq 0$ for each $i$ and ${ }^{0}\left(\sum_{i=1}^{\omega} \beta_{i}\right)<+\infty$. Let $f: Y \rightarrow{ }^{*}[-n, n]$ for some $n \in N$ be internal, and let $\nu(A)=\Sigma_{y_{i} \in A} \beta_{i}$ for each internal set $A \subset$ $Y$. Then there is a unique extension $\mu$ of ${ }^{0} \nu$ to the smallest (external) $\sigma-a l$ gebra containing all internal subsets of $Y$, and

$$
\int_{X}{ }^{0} f(y) d \mu(y) \simeq \sum_{i=1}^{\omega} f\left(y_{i}\right) \beta_{i}
$$

The author is indebted to Professor L. C. Moore, Jr. for calling to his attention the following corollary to Theorems 1 and 3.

Proposition 2. Assume $\mu(X)<+\infty$. If $g: X \rightarrow R \cup\{+\infty,-\infty\}$ is $\prod_{-m e a-}$ surable, then there is an $f: X \rightarrow{ }^{*} R$ which is $Q$-measurable such that of $=$ $g \mu$-almost everywhere.

Proof. We assume that $g$ is bounded. The general case follows by obtaining *finite sequences $\left\{A_{n} \in \mathbb{G}: 1 \leq n \leq \omega\right\}$ and $\left\{f_{n}: 1 \leq n \leq \omega\right\}$ such|that: (i) $A_{n} \cap A_{m}=\varnothing$ if $n \neq m$, (ii) each $f_{n}$ is $\mathcal{G}_{\text {-measurable with support } A_{n} \text { and }}$ ${ }^{0} f_{n}(x)^{n}=g(x)$ for $\mu$-almost all $x \in A_{n}$ when $n \in N$, and (iii) if for $n \in N$ we let $B_{n}=g^{-1}[\{+\infty,-\infty\} \cup[-n, n]]$, then $\bigcup_{i=1}^{n} A_{i} \subset B_{n}$, and $\mu\left(B_{n}-\bigcup_{i=1}^{n} A_{i}\right)<$ $1 / n$. Here $f=\sum_{i=1}^{\omega} f_{i}$.

Assuming $0 \leq g \leq M$ on $X$, let $\left\{g_{n}: n \in N\right\}$ be an increasing sequence of M-measurable simple functions such that $0 \leq g-g_{n} \leq 1 / n$ for each $n \in N$.

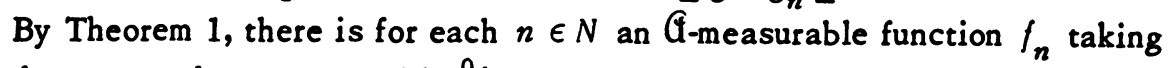
the same values as $g_{n}$ with ${ }^{0} f_{n}=g_{n} \mu$-a.e. If $n<m$ in $N$,

$$
\int_{X}^{0}\left|f_{n}-f_{m}\right| d \nu=\int_{X}\left|{ }^{0} f_{n}-{ }^{0} f_{m}\right| d \mu=\int_{X}\left|g_{n}-g_{m}\right| d \mu \leq \frac{1}{n} \mu(X) .
$$

There exists, therefore, an $\mathbb{G}$-measurable function $f_{\omega^{:}} X \rightarrow{ }^{*}[0, M]$ such that for each $n \in N, \int_{X}\left|f_{n}-f_{\omega}\right| d \nu<(\mu(X)+1) / n$. Thus

$$
\int_{X}\left|g-{ }^{0} f_{\omega}\right| d \mu \leq \int_{X}\left|g-{ }^{0} f_{n}\right| d \mu+\int_{X}\left|{ }^{0} f_{n}-{ }^{0} f_{\omega}\right| d \mu \leq \frac{2}{n}(j(X)+1)
$$

for each $n \in N$; i.e., $g={ }^{0} / \omega$-a.e.

3. Applications to probability theory.

Example 4 (Coin tossing). Fix $\omega \epsilon^{*} N-N$ and let $X$ be the set of all 
internal sequences of zeros and ones of length $\omega$; the internal cardinality of $X,|X|$, is $2^{\omega}$. Let $\mathcal{G}$ be the class of internal subsets of $X$, and for each $A$ $\epsilon \in \mathbb{A}$ let $\nu(A)=2^{-\omega}|A|$. Then $(X, \mathscr{G}, \nu)$ is the internal probability space for the experiment of tossing a fair coin $\omega$ times. Any event depending on only a standard finite number of coin tosses corresponds to a set $A \in \mathbb{G}$, and $\nu(A)$ is the usual probability for such an event. Since $\pi$ is the smallest $\sigma$-algebra in $X$ containing $\mathscr{G}, \pi$ contains the $\sigma$-algebra corresponding to all the "standard events" of coin tossing. In general, standard events correspond to those sets $B \in \mathbb{M}$ such that if the sequence $\left\{x_{i}\right\} \in B,\left\{y_{i}\right\} \in X$ and for all $i$ less than some infinite $\gamma \in{ }^{*} N-N$ we have $x_{i}=y_{i}$, then $\left\{y_{i}\right\} \in B$.

Consider, for example, $A_{n}$ as the event "the first $n-1$ tosses are tails, the $n$th toss is a head." If $\omega$ is even, the set $A=\bigcup_{n=1}^{\omega / 2} A_{2 n}$ in $G$ corresponds to getting a head, the first one occurring at an even numbered toss in $\omega$-tosses. Moreover,

$$
\nu(A)=\sum_{n=1}^{\omega / 2} \frac{1}{2^{2 n}}=\frac{1}{3}-\frac{1}{3 \cdot 3^{\omega}}
$$

and $\mu(A)=1 / 3$. On the other hand, the set $B=\bigcup_{n=1 ; n \in N}^{\infty} A_{2 n}$ in $\mathbb{M}$ corresponds to the standard event of getting at least one head, the first one occurring at an even numbered toss in an infinite number of tosses. Of course, $\mu(B)=1 / 3$. It is by working with sets such as $B$, sets which correspond to standard events, that one can use $(X, \Re, \mu)$ as a "standard" model for coin tossing.

Example 5 (Poisson processes). Fix $\omega \epsilon^{*} N-N$ and $\lambda>0$ in $R$, and let $\gamma$ be the greatest element of ${ }^{*} N$ with $\gamma \leq \lambda \omega$. Divide the interval $[0, \omega)$ ințo $\omega^{2}$ intervals $[0,1 / \omega),[1 / \omega, 2 / \omega), \ldots,\left[\left(\omega^{2}-1\right) / \omega, \omega\right)$, and let $X$ denote all $\omega^{2 \gamma}$ ways that $\gamma$ "balls" can be put into the $\omega^{2}$ "boxes" $[k / \omega$, $(k+1) / \omega)$. That is, $X$ is the set of all internal sequences $\left\{x_{i}\right\}$ with $1 \leq i \leq$ $\gamma$ and $1 \leq x_{i} \leq \omega^{2}$ for each $i$. Let $\mathcal{G}$ consist of all internal subsets of $X$ and for each $A \in \mathbb{Q}$, let $\nu(A)=|A| / \omega^{2 \gamma}$. The probability space $(X, \mathbb{M}, \mu)$ can be used as a model for standard Poisson processes such as radioactive decay, incoming telephone calls, etc. If, for example, $k \in N$ and $T$ is a finite interval of length $t$, the following version of the well-known calculation using the binomial distribution (see [3, p. 69]) gives the probability $\nu(A)$ of the internal event "There are exactly $k$ balls in $T$ ": We assume for simplicity that $\omega=$ $\eta$ ! for some $\eta \epsilon^{*} N-N$ and that $\lambda$ and the endpoints of $T$ are standard rational numbers. Then $\gamma=\omega \lambda$ and there are exactly $t \omega$ of the $\omega^{2}$ intervals inside $T$. The probability of putting any one of the $\gamma$ balls in $T$ is $t \omega / \omega^{2}=$ 
$t / \omega=\lambda t / \gamma$. Therefore

$$
\begin{aligned}
\nu(A) & =\frac{\gamma !}{(\gamma-k) ! k !} \cdot\left(\frac{\lambda t}{\gamma}\right)^{k} \cdot\left(1-\frac{\lambda t}{\gamma}\right)^{\gamma-k} \\
& =\frac{(\lambda t)^{k}}{k !} \cdot \frac{\gamma !}{\gamma^{k}(\gamma-k) !} \cdot\left(1-\frac{\lambda t}{\gamma}\right)^{\gamma} \cdot\left(1-\frac{\lambda t}{\gamma}\right)^{-k} \\
& \simeq \frac{(\lambda t)^{k}}{k !}\left(1-\frac{\lambda t}{\gamma}\right)^{\gamma} \simeq \frac{(\lambda t)^{k}}{k !} e^{-\lambda t}=\mu(A) .
\end{aligned}
$$

Thus, $\mu(A)$ is the value at $k$ of the Poisson distribution with parameter $\lambda t$.

Now for each $x \in X$, order the balls by the order in which they fall in the line ${ }^{*} R$, so that for $1 \leq i<\gamma$ we have the $i$ th ball $b_{i} \leq b_{i+1}$, with equality holding if and only if $b_{i}$ and $b_{i+1}$ are in the same interval $[k / \omega,(k+1) / \omega)$. Again fix $t>0$, a standard rational number, and fix $j>0$ and $k \geq 0$ in $N$. Given an interval endpoint $t_{0}=0,1 / \omega, 2 / \omega, \ldots,\left(\omega^{2}-1\right) / \omega$, let $C_{t_{0}}$ be the event " $b_{j} \in\left[t_{0}, t_{0}+1 / \omega\right)$ ", and let $D_{t_{0}}$ be the event "For $j+1 \leq i \leq j+k_{\text {, }}$ $b_{i} \in\left[t_{0}, t_{0}+t\right)$ and $b_{j+k+1} \notin\left[t_{0}, t_{0}+t\right)_{0}$ " Let $\gamma^{\prime}=\gamma-j$. Given $C_{t_{0}}$, the conditional probability of getting a given ball of the remaining $\gamma^{\prime}$ balls in $\left[t_{0}, t_{0}+t\right)$ is

$$
\frac{t \omega}{\omega^{2}-t_{0} \omega}=\frac{t}{\omega-t_{0}}=\frac{\lambda t}{\gamma-t_{0} \lambda}=\frac{\lambda t}{\gamma^{\prime}+j-t_{0} \lambda} .
$$

Thus for all finite $t_{0}$, and therefore for all $t_{0} \leq \tau$ for some infinite $\tau$, we have the conditional probability

$$
\nu\left(D_{t_{0}} \mid C_{t_{0}}\right)=\frac{\gamma^{\prime} !}{\left(\gamma^{\prime}-k\right) ! k !} \cdot\left(\frac{\lambda t}{\gamma^{\prime}+j-t_{0}^{*} \lambda}\right)^{k} \cdot\left(1-\frac{\lambda t}{\gamma^{\prime}+j-t_{0} \lambda}\right)^{\gamma^{\prime}-k} \simeq \frac{(\lambda t)^{k}}{k !} e^{-\lambda t} \text {. }
$$

On the other hand, $\Sigma_{t_{0}<r} \nu\left(C_{t_{0}}\right) \simeq 1$, and so $\Sigma_{t_{0}<\tau} \nu\left(D_{t_{0}} \mid C_{t_{0}}\right) \cdot \nu\left(C_{t_{0}}\right) \simeq$

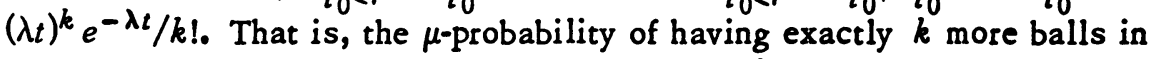
the interval of length $t$ after the $j$ th ball is $\left(\lambda_{t}\right)^{k} e^{-\lambda t} / k !$. This gives an elementary proof that for these "stopping times", i.e., the time of the $j$ th event, Poisson processes have the strong Markov property. Since

$$
\sum_{k=0 ; k \in N}^{\infty} \frac{(\lambda t)^{k}}{k !} e^{-\lambda t}=e^{\lambda t} \cdot e^{-\lambda t}=1,
$$

the $\mu$-probability of having only a finite number of balls in any finite interval $[0, t)$ is 1. Moreover, since $\lim _{t \rightarrow 0} e^{-\lambda t}=1$, the $\mu$-probability of having ball $b_{j+1}$ infinitely close to $b_{j}$ is 0 , and this being true for each $j \geq 1$ in $N$, it follows that the $\mu$-probability of having two balls in the same monad is 0 . 
Let $R^{+}$be the nonnegative real numbers and $B$ the Borel sets in $R^{+}$. For each $t \epsilon^{*} R^{+}$and $x \in X$, let $f(t, x)$ be the number of balls in $[0, t]$, where we assume now that any ball in the box $[k / \omega,(k+1) / \omega)$ is at $k / \omega$. Although $f(t, x)$ is $* \mathfrak{B} \times \mathbb{Q}$-measurable on ${ }^{*} R^{+} \times X$, we would like to restrict the values of $t$ to standard values. That we can do so is a consequence of the following general result.

Theorem 4. Assume that $\mathbb{Q}$ is an internal $\sigma$-algebra (in the nonstandard sense) in $X$. Let $f:{ }^{*} R^{+} \times X \rightarrow{ }^{*} R$ be an internal ${ }^{*} \mathcal{B} \times \mathbb{G}$-measurable function such that $f(t, x)$ is an increasing function of $t$ for each $x \in X$. Let $g: R^{+} \times$ $X \rightarrow R \cup\{+\infty\} \cup\{-\infty\}$ be defined by setting $g(s, x)=\sup _{t \simeq s} 0 f(t, x)$ for each $x \in X$. Then $g(s, x)$ is an increasing and right continuous function of $s$ for each $x \in X$, and $g$ is $B \times M-m e a s u r a b l e$ on $R^{+} \times X$.

Proof. Clearly, $g(s, x)$ is an increasing function of $s$ for each $x \in X$. Fix $x \in X$ and $s \in R^{+}$, and let $a=g(s, x)$. Assume that $g(\cdot, x)$ is not right continuous at $s$. Then $\exists \epsilon>0$ in $R$ so that for each $n \in N$ there is a $t \epsilon^{*} R$ with $s<t<s+1 / n$ such that $f(t, x)-a \geq \epsilon$. It follows that there are an $\omega \epsilon$

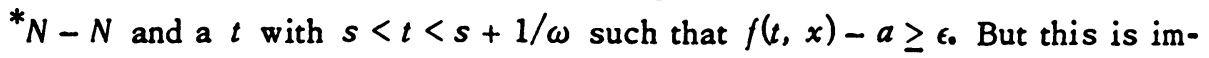
possible since ${ }^{0} f(t, x) \leq a$. Thus $g(\cdot, x)$ is right continuous at $s$.

To show that $g$ is $\mathbb{B} \times \mathbb{M}$-measurable, we let $h_{a}$ be the function defined on $X$ for a given $a \in R$ by setting $h_{a}(x)=\inf \left\{t \in *^{*} R^{+}: f(t, x) \geq a\right\}$. Then $h_{a}$ is $\mathbb{Q}_{\text {-measurable since }}\left\{x: h_{a}(x) \geq 0\right\}=X$ and for any $\beta>0$ in ${ }^{*} R$,

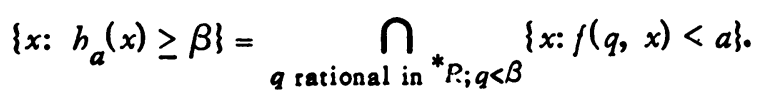

It follows from Theorem 2 that ${ }^{0} h_{a}$ is $\mathbb{M}$-measurable on $X$, and thus $\{(s, x) \epsilon$

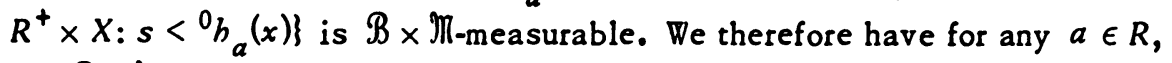
the $\Re \times M_{\text {-measurable set }}$

$$
\begin{aligned}
\bigcup_{n=1 ; n \in N}^{\infty}\left\{(s, x) \in R^{+}\right. & \left.\times X: s<{ }^{0} h_{a-1 / n}(x)\right\} \\
& =\bigcup_{n=1 ; n \in N}^{\infty}\left\{(s, x) \in R^{+} \times X: \forall t \simeq s, f(t, x)<a-1 / n\right\} \\
& =\left\{(s, x) \in R^{+} \times X: g(s, x)<a\right\} .
\end{aligned}
$$

\section{REFERENCES}

1. A. Bernstein and P. A. Loeb, A nonstandard integration theory for unbounded functions, Victoria Sympos. on Nonst andard Analysis, Lecture Notes in Math., -vol. 369, Springer-Verlag, New York, 1974, pp. 40-49. 
2. W. K. Feller, An introduction to probability theory and its applications. Vol. I, 2nd ed., Wiley, New York; Chapman and Hall, London, 1957. MR 19, 466.

3. P. G. Hoel, S. Port and C. Stone, Introduction to probability theory, Houghton Mifflin, Boston, Mass., 1971.

4. P. A. Loeb, $A$ nonstandard representation of measurable spaces, $L_{\infty}$ and $L_{\infty}^{*}$ Contributions to Nonstandard Analysis, edited by W. A. J. Luxemburg and A. Robinson, North-Holland, Amsterdam, 1972, pp. 65-80.

5. - A nonstandard repres entation of Borel measures and O-finite measures, Victoria Sympos. on Nonstandard Analysis, Lecture Notes in Math., vol 369, Springer Verlag, New York, 1974, pp. 144-152.

6. W. A. J. Luxemburg, A general theory of monads, Applications of Model Theory to Algebra, Analysis and Probability (Internat. Sympos., Pasadena, Calif., 1967), Holt, Rinehart and Winston, New York, 1969, pp. 18-86. MR 39 \#6244. $\# 5680$.

7. A. Robinson, Non-standard analysis, North-Holland, Amsterdam, 1966. MR 34

8. H. L. Royden, Real analysis, Macmillan, New York, 1968.

DEPARTMENT OF MATHEMATICS, UNIVERSITY OF ILLINOIS, URBANA, ILLINOIS 61801 